CERN/PS 97-49(OP)

7 July 1997

\title{
EMITTANCE PRESERVATION IN THE PS COMPLEX
}

B. Autin, L. Giulicchi, A. Jansson, M.Lindroos, A. Lombardi, M. Martini, K. Schindl

\begin{abstract}
As the LHC injectors have to provide bright beams, all the potential sources of emittance blowup must be eliminated. One such source arises from the mismatch of the betatron focusing at the interface of a transfer line with a circular machine. Measurements and corrections of this effect have been performed in the line downstream of the linac where space charge plays an important role and between the booster and the PS ring where four beams are recombined and have to be matched simultaneously.
\end{abstract}

(Presented at the PAC97 meeting of the American Physical Society) 


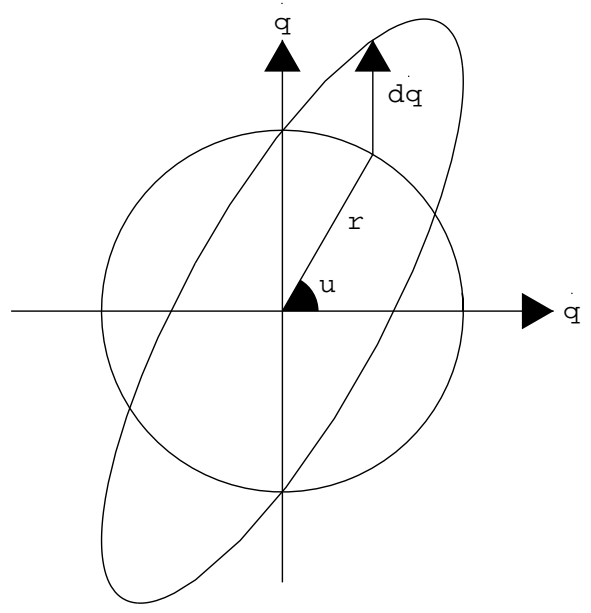

Figure 1: Effect of a quadrupolar error in the normalized phase plane.

\section{INTRODUCTION}

Emittance preservation is a subject of concern for many high energy machines. In the case of the CERN PS complex, the most severe tolerances are imposed by the LHC beam. A program has therefore been set-up to investigate the sources of beam blow-up and to measure and correct the emittance with the required precision. The LHC injector chain consists of a total of four accelerators: linac, booster, PS and SPS. The tolerance on the beam size blow-up measured at $1 \sigma$ is smaller than $20 \%$ at the end of each of the accelerators. In a given linear machine and at low space-charge, the normalized emittance is a constant of the motion as far as external fields are concerned and can only be affected by instabilities. At the interface between two machines, the emittance may increase if the injection trajectory is not tangent to the closed orbit and if the focusing of the transfer line does not match the focusing of the accelerator. The first mechanism produces an oscillation of the centre of gravity of the beam; this is the problem of coherent oscillations [1]. The second effect produces a beam envelope oscillation and is the subject of this paper.

\section{THEORETICAL BACKGROUND}

A focusing error related to a quadrupole field can be treated within the general framework of first order perturbations. The position $x$ and its derivative with respect to the curvilinear abscissa $s$ are replaced by new normalised variables

$$
q=\frac{x}{\sqrt{\beta}} \quad \dot{q}=\frac{d q}{d \mu}
$$

where $\beta$ is the $\beta$-function at the point of abscissa $s$ and $\mu$ the betatron phase advance at the same point. The betatron motion is thus described by a harmonic oscillator whose motion in phase space is a circle of radius the square root of the emittance, $\varepsilon$. A magnet of infinitesimal length $d s$ and of focusing error $k$ at a point of phase $\mu$ produces a kick

$$
d \dot{q}=k \beta q d s
$$

This kick changes the betatron emittance, considered not as the area of the ellipse but as a measure of the oscillation amplitude, and the phase of each particle by the quantities

$$
d \varepsilon=2 \dot{q} d \dot{q} \quad d \mu=\frac{q d \dot{q}}{\varepsilon}
$$

whose detailed form can be written

$$
\frac{d \varepsilon}{\varepsilon}=\sin 2 \mu \mathrm{k} \beta \mathrm{ds} \quad \mathrm{d} \mu=\frac{1+\cos 2 \mu}{2} \mathrm{k} \beta \mathrm{ds}
$$


After the kick, the circle is transformed into an ellipse (see Fig. 1). The total focusing error is given by the integral over $s$ of the above expressions. The term independent of $\mu$ in the phase perturbation is not relevant in this context because it corresponds to a global rotation of all the particles and does not change the shape of the ellipse which is characterized by the vector:

$$
V=\int_{0}^{L}\left(\begin{array}{c}
\sin 2 \mu \\
\cos 2 \mu
\end{array}\right) k \beta d s
$$

where $L$ is the length of the transfer line. The quadrupoles act in the horizontal and vertical planes simultaneously and the focusing error is corrected by minimizing the norm of the four-component mismatch vector

$$
W=\int_{0}^{L}\left(\begin{array}{c}
\beta_{h} \sin 2 \mu_{\mathrm{h}} \\
\beta_{h} \cos 2 \mu_{\mathrm{h}} \\
-\beta_{v} \sin 2 \mu_{\mathrm{v}} \\
-\beta_{v} \cos 2 \mu_{\mathrm{v}}
\end{array}\right) k d s
$$

The derivation of the vector $W$ has been based on the classical action-angle perturbation theory. The mismatch can be measured [2] and expressed in terms of the Courant Snyder invariants characterized by $\beta$ and $\alpha$ functions. The contribution of $m$ discrete and local gradient errors, $k_{j}$, in quadrupoles (of length $l_{j}$ at $\mu_{j}$ ) to the $\alpha$ and $\beta$ functions at a monitor $(i)$ can be written as the sum over the $m$ correctors

$$
\left(\begin{array}{c}
\frac{\Delta \beta}{\beta_{i}} \\
\Delta \alpha_{i}-\alpha_{i} \frac{\Delta \beta}{\beta_{i}}
\end{array}\right)=\sum_{j=1}^{m}\left(\begin{array}{l}
k_{j} l_{j} \beta_{j} \sin 2\left(\mu_{\mathrm{i}}-\mu_{\mathrm{j}}\right) \\
k_{j} l_{j} \beta_{j} \cos 2\left(\mu_{\mathrm{i}}-\mu_{\mathrm{j}}\right)
\end{array}\right)
$$

where Eq. (7) can be derived from of Eq. (6).

The correction procedure, called Micado [3], is based on an iterative reduction of the norm of the residual vector. The correction matrix $A$ has as many columns as correctors; each column is the mismatch vector $W$ produced by a unit error; this error can be a focusing strength expressed in $m^{-} 2$ or a current in Ampere. $b$ is the measured mismatch vector at the interface between the transfer line and the machine. $x$ is the unknown correction vector; at the first iteration, it contains one component attached to the best corrector; at the $n$-th iteration, the $n-1$ previous correctors are maintained, the best new corrector is chosen and all the corrections are recalculated. The procedure is stopped when the norm is limited by experimental errors.

\section{LINAC LINE}

The transfer line between linac and booster can be conceptually divided in three parts: the first part (from the output of the linac to the first bending) is space-charge dominated as the beam coming out of the linac is still strongly bunched (some $10^{\circ}$ at $200 \mathrm{MHz}$ ); the second part (between the two bendings ) is in a medium space-charge regime, and the third part (up to the last of two emittance measuring lines) is in a low space-charge regime as the beam is almost completely debunched. The matched input ellipse to the booster has been back-traced to the emittance measuring line defining slit where the matching is monitored. The matching is performed by the last four quadrupoles. The purpose of our work, which goes under the name of the Automated Beam Shaping (ABS) project, is the automatic correction of the transverse ellipse parameters at the defining slit.

The application of Micado requires the evaluation of the mismatch vector and correction matrix. The mismatch vector is derived by measuring the emittance of two consecutive cycles to acquire data on the horizontal and vertical planes and comparing it to the target value. The correction matrix represents the sensitivity of the mismatch vector components to small changes in quadrupole input currents.

Considering the limited accuracy introduced by space-charge effects, the evaluation of the quadrupole matrix is one of the most critical points in the application of the ABS procedure.

The evaluation of the matrix has been performed using two methods: the first method is to compute the sensitivity matrix using a beam dynamics model implemented in Trace 3D software tool [4] , the second by experimental measurements.

The matrix has been built by evaluating the mismatch vector components for $\pm 5 \%$ change in the quadrupole currents, fitting them with a parabolic function and finally computing the derivative on the reference current value. In Fig. 2 the theoretical and the measured norm of the mismatch vector are shown. The norm has been reduced by a factor of five using four correctors. The correctors may be less efficient if the initial mismatch is big. Tests with a norm of the order of unity, clearly not within the scope of first order perturbations, could be nevertheless reduced by a factor of two using two correctors. 

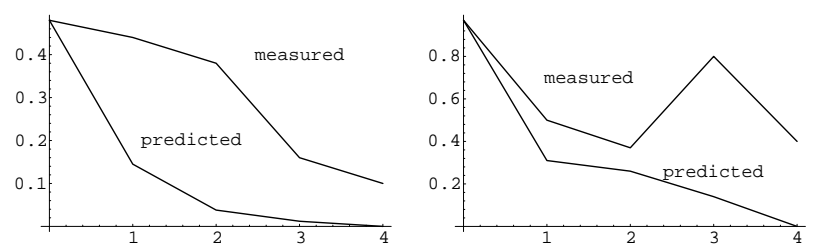

Figure 2: Theoretical and measured norms of the combined total mismatch vector in the linac line after compensation with $1,2,3$ and 4 correctors.

\section{TRANSFER BETWEEN BOOSTER AND PS}

\subsection{Layout of the transfer line}

To fill the CPS, the four PS booster beams are ejected sequentially. The present recombination scheme results in non-negligible differences in optical parameters for the four rings at the beginning of the common beam-line section [5]. A solution exists for curing these differences and it is likely to be implemented in the near future. After the recombination, the beams ideally follow the same trajectory. From here the beam can be sent to different destinations, either ISOLDE, CPS or the PSB measurement line. The latter is equipped with three SEM-grids for transverse optics measurements.

\subsection{Theoretical model}

As space charge at $1 \mathrm{GeV}$ is negligible for optics calculations an analytical package, BeamOptics [6], has been used for the modelling. An advantage with this analytical program is that it can directly generate the the correction matrices.

The inherent difference in the optics between the four booster rings gives a lower limit to the PS injection mismatch. If we try to minimize the mismatch for one of the beams, this will result in increased mismatch in the other beams. A better approach is to try to distribute this effect over the four beams as evenly as possible. This can be achieved by putting the correction matrices for the individual beams together in a global matrix.

\subsection{Experimental results}

The correction method has been tested with positive results in the Booster measurement line. The measurements were made in the horizontal plane only, since the quadrupole settings for the line have to be different for horizontal and vertical measurements. The vertical correction was set to zero, $\mathrm{i}$ e the vertical mismatch vector was constrained not to change. The results for the correction, Fig. 3, show a significant reduction of the horizontal mismatch. Tests have also been performed in the PS ring, using
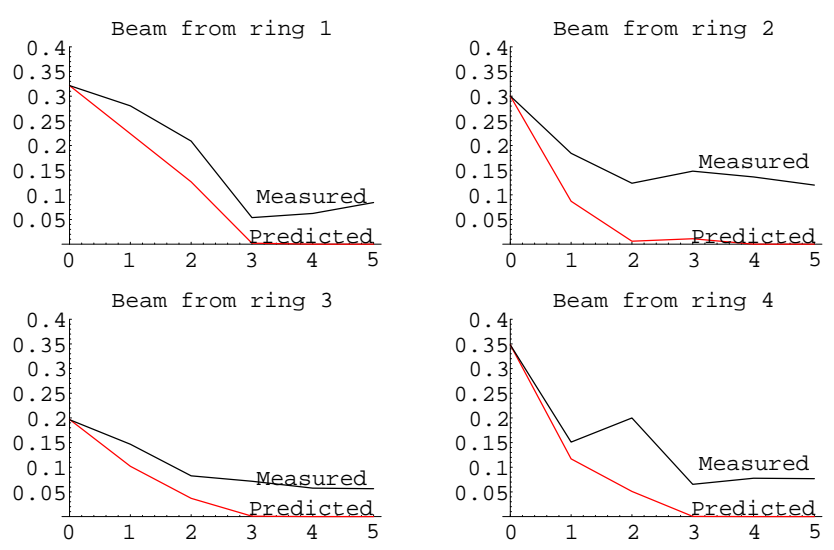

Figure 3: Results in the PSB Measurement line, using individual matrices. The absolute value of the horizontal mismatch vector is plotted as a function of number of correctors used.

three SEM-grids and a beam stopper. In this case both horizontal and vertical Twiss parameters can be measured with the same optics and it is possible to check if the correction is effective in both planes or not. The results are shown in Fig. 4. 


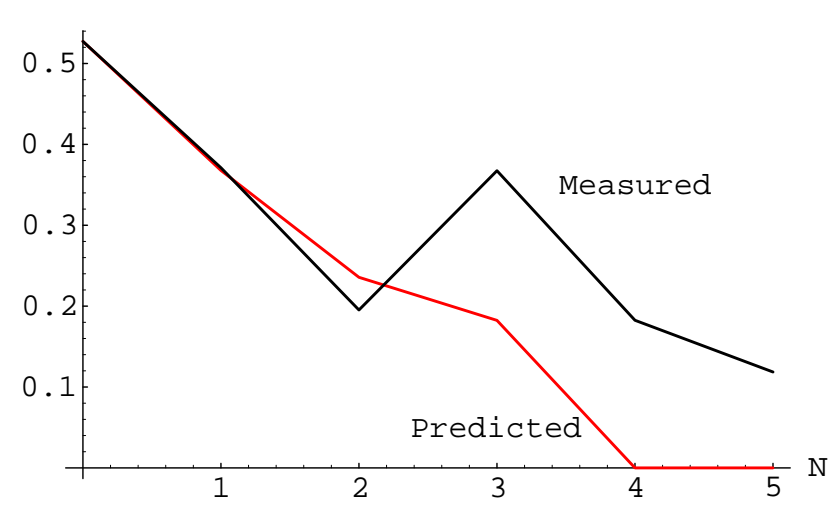

Figure 4: Results in the PS ring using up to 5 corrcetors in the booster-PS transfer line. The measurement was performed using beam from PSB ring three. The absolute value of the total mismatch vector is plotted as a function of number of correctors used.

\section{CONCLUSIONS}

The good general agreement between theory and measurements in the linac line, the booster measurement line and in the CPS ring confirms the validity of the approach to the problem of mismatch minimization. In the cases tackled, the initial mismatch was rather small, but there are indications that the procedure may diverge for larger values. It is possible to compensate for this by an iterative procedure where the initial mismatch is reduced with one or two correctors. The measurement is then repeated followed by new corrections. First tests of such a procedure show significant improvement of the convergence.

It should be stressed that the results presented here have been achieved using existing measurement software, off-line calculated corrections, which were manually transferred to the quadrupoles. This procedure will be replaced in the near future by a dedicated, but generic software concerning machine part and type of correction. The method can then be made available for the daily work of maintaining the quality of the operational beams. Furthermore, the present very time consuming iterative procedure can be fully tested.

\section{ACKNOWLEDGEMENTS}

Many thanks to the TRIUMF laboratory for supplying the laminated quadrupoles for the linac line. Many thanks also to Michel Arruat and Olav Tungesvik of our division for prompt and highly reliable software support.

\section{References}

[1] B. Autin, V. Ducas, A. Lombardi, M. Martini, E. Wildner: 'Automated beam optics correction for emittance preservation', Proc. LHC Workshop on LHC Injectors published by Particle Accelerators (1997).

[2] M. Martini, H. Schonauer: 'Emittance measurement', Proc. LHC Workshop on LHC Injectors published by Particle Accelerators (1997).

[3] B. Autin and Y. Marti, 'Closed orbit correction of AG machines using a small number of magnets', CERN ISR-MA/73-17.

[4] K. R. Crandall, 'TRACE 3-D documentation', Los Alamos Accelerator Code Group, LA-UR-90-4146

[5] A. Jansson and M. Lindroos: 'Mismatch between the PSB and CPS due to the present vertical recombination scheme', CERN/PS 97-14, Proc. LHC Workshop on LHC Injectors published by Particle Accelerators (1997).

[6] B. Autin, T. d'Amico, M. Martini, E. Wildner, 'Analytic lattice design with BeamOptics', These Proceedings. 